\title{
Vivencias de migrantes mexicanos sobre estados emocionales experimentados durante su proceso migratorio y el consumo de alcohol y drogas
}

\section{Mexican migrants experiences about emotions lived during their migration process, with alcohol and drugs consumption}

Teresa Margarita Torres López, * José Luis López López, **

Miguel Alfonso Mercado Ramírez ${ }^{* * *}$ y Amparo Tapia Curiel ${ }^{* * * *}$

Resumen

El objetivo es analizar las vivencias de migrantes mexicanos en México y en Estados Unidos en torno a diversos estados emocionales que experimentan durante la migración, acompañada del consumo de alcohol y drogas. Se realiza a partir de un estudio etnográfico, con entrevistas a profundidad en 19 individuos en México y 19 en Estados Unidos. El análisis fue fenomenológico. Los participantes señalaron diferentes estados emocionales negativos ligados a diversos patrones relacionados con el consumo de alcohol y drogas, en las diferentes fases de la migración.

Palabras clave: emociones, alcohol, drogas, migrantes, apoyo social.

Recibido el 21 de noviembre de 2013.

Aprobado el 9 de diciembre de 2013.

\begin{abstract}
The objective of this study was to analyze migrant experiences in México and United States of America (USA) about emotions lived during the migration process along with alcohol and drugs consumption. It was an ethnographic study with depth interviews, 19 in Mexico and 19 in UsA. The analysis was phenomenological. The participants pointed out negative and depressive emotions, in connection with different consume patterns of alcohol and drugs, along the migration phases.

Keywords: emotions, alcohol, drugs, migrants, social support.
\end{abstract}

* Doctorado en Antropología Social y Cultural. Departamento de Salud Pública. Universidad de Guadalajara, México. Correo electrónico: tere.torres.cucs@gmail.com

** Doctorado en Ciencias de la Salud, Orientación Sociomédica. Departamento de Salud Pública. Universidad de Guadalajara, México. Correo electrónico: jose.luis. lopez@cucs.udg.mx

***Maestro de Ciencias de la Salud Pública. Departamento de Salud Pública. Universidad de Guadalajara, México. Correo electrónico: ortomiguel@hotmail.com ****Doctorado en Ciencias de la Salud, Orientación Sociomédica. Departamento de Salud Pública. Universidad de Guadalajara, México. Amp_tap_cur@hotmail.com 


\section{Introducción}

Para la Comisión de Salud Fronteriza México-Estados Unidos, los censos del año 2000 de México y Estados Unidos de América informaron la existencia de casi 13 millones de habitantes en la región fronteriza, divididos equitativamente entre México (6.4 millones) y Estados Unidos (6.6 millones) (Comisión de Salud Fronteriza México-Estados Unidos, 2003, p. 9). En 2004 la Iniciativa de Salud México-Estados Unidos reportó que cuatro estados fronterizos de Estados Unidos estuvieron entre los 13 estados con más de 100000 inmigrantes mexicanos: Nuevo México (111 049), Arizona (618 105), Texas (2 356702 ) y California (4 026 219). Entre los cuatro estados en 2005, se contaban más de siete millones de inmigrantes mexicanos residentes (OPS, 2007). ${ }^{1}$

Entre las múltiples necesidades de la población inmigrante, están las relacionadas con los problemas de salud. Según la Organización Panamericana para la Salud (OPS), estos problemas son similares en cada lado de la frontera y afectan a poblaciones iguales (ops, 2007). Por su parte, la Agenda Bilateral del Programa Frontera Saludable 2010 señaló problemas importantes para ambos países, entre los que destacan el tabaquismo, el abuso de sustancias como alcohol y drogas, las enfermedades del corazón y gastrointestinales, la nutrición y obesidad, la actividad física, la capacitación para el bioterrorismo y la investigación en salud y ciencias de la conducta (Comisión de Salud Fronteriza México-Estados Unidos, 2003, p. 5).

De particular importancia se encuentran los problemas de salud mental, derivados de los riesgos y el estrés relacionados con la migración. Salgado (1996) señala que las diferentes fases del fenómeno (antes, durante y después) implican la presencia de situaciones estresantes que, aunado a otros riesgos, pueden derivar en daños a la salud física y mental.

La migración es un fenómeno complejo, en el cual las personas que migran pasan por etapas de ajuste frente a diversos estresores relacionados con la preparación, el trayecto y la adaptación a una nueva cultura (Bhugra, 2004). En la etapa previa a la migración se definen las motivaciones para realizar el traslado, pero tiene un peso importante si se realiza de

\footnotetext{
${ }^{1}$ Agradecemos a los migrantes y sus familias que cooperaron en la elaboración de este estudio. También agradecemos al Programa de Investigación en Migración y Salud (PIMSA) por el apoyo financiero del proyecto en la Convocatoria 2009-10.
} 
forma voluntaria o forzada, o bien, en condición legal o ilegal. En caso de ser ilegal, la experiencia de cruzar la frontera suele ser sumamente estresante, ya que implica fatiga física y psicológica, además del riesgo de experimentar situaciones de violencia, extorsión, deportación, encarcelamiento e incluso de muerte. En la fase de estancia surgen cambios conductuales y actitudinales, propios de la aculturación, lo que causa estrés psicológico asociado a la reorganización de habilidades cognoscitivas y adaptativas. A estas situaciones se adiciona el duelo ante la pérdida de las relaciones familiares y de amistad, de bienes materiales y apoyo social (Salgado, 1996).

Los procesos de ajuste se asocian a diversos estresores; entre éstos se encuentran los relacionados con la situación económica, el acceso a fuentes de empleo, las relaciones maritales y parentales, así como los cambios culturales (Salgado, 2002).

Económicamente, un bajo nivel de escolaridad, una condición ilegal, el miedo a ser deportado, o el dominio insuficiente o nulo de un nuevo idioma, pueden llevar a los migrantes a desempeñar trabajos con salarios bajos o en condiciones adversas; esto es más patente con los trabajadores de campo, donde se observa hacinamiento y vulnerabilidad en general (Holmes, 2006).

Si se ha migrado en forma individual o familiar, entonces las relaciones familiares se viven de forma diferente. La migración individual puede presentar distanciamiento físico y emocional; extrañar el ambiente familiar, sentir culpabilidad por familiares que permanecen en México, también sufrir angustia por falta de apoyo y por las tensiones que se generan a través de las relaciones a larga distancia (Bhugra, 2004). Cuando se emigra con la familia se pueden dar cambios en las formas tradicionales de relación marital, como: la pérdida de autoridad del hombre cuando la mujer comienza a trabajar y se independiza, el control de los ingresos económicos, las decisiones sobre la educación de los hijos y las preferencias religiosas (Salgado, 1996).

Culturalmente se pueden manifestar situaciones de tensión por desconocer la cultura del nuevo país, agravadas, además, por un dominio insuficiente del idioma. Los cambios en el estilo de vida y no percibir el cumplimiento de las expectativas personales y familiares, así como experimentar situaciones de discriminación, pueden afectar la identidad y los valores de prestigio y dignidad de las personas (Salgado, 1996, 2002). Estas 
circunstancias favorecen la presencia de estados emocionales que afectan la salud mental.

Para la ops (2009), los patrones en el consumo de drogas en México han cambiado; existe un aumento en el consumo de heroína, cocaína y estimulantes, sobre todo en poblaciones de la zona fronteriza con Estados Unidos. El consumo de mariguana ha descendido, aunque ésta es la droga ilegal más usada en México. En un estudio realizado en las ciudades de Tijuana y Monterrey (México), los datos corroboran que la primera preferencia es el consumo de la mariguana, la segunda, de la cocaína, y la tercera, de los estimulantes y tranquilizantes (Rojas, Fleiz, Villatoro, Gutiérrez y Medina-Mora, 2009).

La Encuesta Nacional de Adicciones (México, 2011) reveló que la región norte tiene las tendencias más altas en consumo de cualquier droga, $2.8 \%$ en 2011 (2.4\% en 2008), mientras que en la región centro esta tendencia fue de $1.7 \%$ y en la región sur de $1.2 \%$. Asimismo, la región norte presenta las mayores tendencias en el consumo de drogas ilegales, 2.3\%, respecto de la región centro con $1.3 \%$ y la región sur con $1.1 \%$ (VillatoroVelázquez, Medina-Mora, Fleiz-Bautista, Téllez-Rojo, Mendoza-Alvarado, Romero-Martínez, Gutiérrez-Reyes et al., 2011, p. 31).

Las tendencias regionales en el consumo de drogas, en el último año, señalan que la región noroccidental (Baja California, Baja California Sur, Sonora y Sinaloa) tiene las prevalencias más elevadas en el consumo de drogas ilegales $(2.8 \%$ ), seguida con $2.4 \%$ por la región nororiental (Tamaulipas, Nuevo León y San Luis Potosí); les siguen la región norcentral (Coahuila, Chihuahua y Durango) y la región occidental (Zacatecas, Aguascalientes, Jalisco, Colima y Nayarit) que presenta la misma tendencia que la media nacional, con 1.5\% (Villatoro-Velázquez et al., 2011, p. 33).

El estudio realizado por Borges, Medina-Mora, Breslau y AguilarGaxiola (2007) señala la asociación entre el consumo de sustancias y la migración hacia Estados Unidos, en mexicanos con diferente experiencia migratoria: a) los que viajan a Estados Unidos en busca de trabajo y permanecen ahí por lo menos tres meses; $b$ ) los que viajan con familiares en ese país; y $c$ ) los que viajan sin experiencia migratoria. Los autores encontraron fuerte asociación entre el abuso de sustancias y la migración hacia Estados Unidos; los migrantes que ya habían laborado en ese país presentaron mayor consumo de alcohol, mariguana, cocaína y otras 
drogas ilícitas, que las personas que no habían vivido esa experiencia. Los investigadores sugieren que este patrón de consumo puede estar ligado a cambios sociales generados por la migración transnacional, tales como mayor acceso a las sustancias en Estados Unidos, tener más medios económicos y el cambio de normas sociales, a los que se aúnan los sentimientos de soledad, aislamiento social y discriminación que se viven en ese país (Borges et al., 2007).

Los estudios de Sánchez-Huesca, Arellánez-Hernández, Pérez-Islas y Rodríguez-Kuri (2006) consideran la relación entre el consumo de sustancias y migración, al comparar a dos grupos de usuarios de droga: uno con experiencia migratoria hacia la frontera norte y/o a Estados Unidos y otro sin experiencia migratoria. Se encontró que aun cuando la mayoría de los usuarios migrantes ya había consumido drogas legales e ilegales en su lugar de residencia, durante la estancia migratoria en la frontera norte o en Estados Unidos los individuos modificaron su patrón de consumo; es decir, estos individuos incrementaron el uso de algunas sustancias o experimentaron con otras y crearon nuevas combinaciones. También hubo aumento en el consumo de metanfetamina, éxtasis, cocaína y crack, así como la experimentación con drogas de uso poco frecuente en México (LSD, PCP, Racki y Artane). La mayor parte de los migrantes hacia la frontera norte usó sustancias depresoras, mientras que el grupo de migrantes hacia Estados Unidos usó más los estimulantes. El predominio en el consumo de estas sustancias, en este último grupo, podría asociarse a la exigencia de mayor rendimiento laboral, para lograr trabajar más horas y, así, ganar la mayor cantidad de dinero en el menor tiempo.

En relación con el consumo de alcohol, García y Gondolf (2004) realizaron estudios con migrantes trabajadores del campo, donde reportan mayor consumo de alcohol en trabajadores solos (solteros, separados, divorciados o viudos), en comparación con quienes viven con sus familias u otros parientes. Alguna de las causas en este consumo se encuentra en las circunstancias que viven, como hacinamiento, aislados en los campos de trabajo, con dificultades para transportarse a los centros urbanos, sin actividades recreativas o con exposición a la soledad y la ansiedad.

En cuanto a la frecuencia y cantidad en el consumo de alcohol, se identifica un patrón de consumo diario entre una y tres cervezas, en los días 
laborables; en fin de semana se llega a consumir más de 24 cervezas. Esto implica, en ocasiones, problemas, peleas y accidentes. Las normas que se establecen en los campos de trabajo pueden influir en estas formas de consumo, ya que en algunos lugares se definen horarios específicos para beber y las cantidades permitidas (García y Gondolf, 2004).

Ahora bien, los estudios anteriores se realizaron con enfoques metodológicos cuantitativos que no daban voz a los directamente implicados, esto es, a los migrantes. Conocer sus puntos de vista, sus vivencias y prácticas ante el problema emocional derivado de su proceso migratorio, así como su posible efecto en el consumo de sustancias, llevó a realizar un estudio titulado "Depresión, ansiedad y consumo de sustancias entre migrantes mexicanos". Éste fue desarrollado, de forma conjunta, por investigadores de la Universidad de Guadalajara (U de G), México, y de la Universidad de El Paso, Texas (UTEP), Estados Unidos, e implicó una aproximación metodológica mixta (cuantitativa y cualitativa).

El trabajo aquí presentado corresponde a los hallazgos obtenidos en la fase cualitativa, con el objetivo de analizar las vivencias de migrantes mexicanos en torno a los diversos estados emocionales que experimentaron durante la migración (tránsito, estancia y retorno) y el consumo de alcohol y drogas entre individuos que, al momento de la investigación, todavía residían en Estados Unidos y otros que ya habían regresado a México. La finalidad de esta investigación es identificar los elementos que permitan fortalecer los programas y las políticas de apoyo a la población migrante (para la que permanece en Estados Unidos, como la que retorna a México).

\section{Métodos: Contextos, participantes y procesos del estudio}

\section{Diseño del estudio}

La base teórica de esta investigación fue el enfoque fenomenológico que se centra en las experiencias e interpretaciones de los fenómenos, por parte de la gente que los vive: la vida cotidiana común y la acción social (Maffessoli, 1997, pp. 149-215). Un estudio etnográfico realizado en dos localidades mexicanas del estado de Jalisco, México, con alto índice de migración a Estados Unidos, y dos localidades del estado de Texas, receptoras de 
migrantes mexicanos (y de otras nacionalidades), entre el mes de abril de 2010 y marzo de 2011.

\section{Contextos de estudio}

En la selección de los contextos se consideró la accesibilidad y el contacto previo con algunos miembros de las comunidades. Además, en el caso de las localidades del estado de Texas, se consideró que $95 \%$ de la población fronteriza vive en 14 pares de ciudades hermanas. La zona metropolitana, formada por las urbes de Ciudad Juárez y El Paso, tiene más de dos millones de habitantes, lo que la convierte en la comunidad fronteriza MéxicoEstados Unidos más grande (ops, 2007).

El trabajo de campo, para el estudio general, en México se realizó en tres comunidades de Jalisco: San Antonio de los Vázquez, Villa Morelos y Ejutla. Las tres poblaciones se caracterizan por su fuerte expulsión migratoria hacia Estados Unidos; sus características culturales son más o menos homogéneas respecto de tradiciones, trabajo y normas de convivencia comunitaria.

La fase cualitativa del estudio sólo se realizó en las localidades de San Antonio de los Vázquez y Villa Morelos; en Ejutla no se pudo aplicar entrevistas a profundidad (sólo cuestionarios) debido al ambiente local en conflicto por problemas relacionados con el narcotráfico y que podrían comprometer a los miembros del equipo de investigación.

San Antonio de los Vázquez es una comunidad ubicada en la región norte del estado de Jalisco, que pertenece al municipio de Ixtlahuacán del Río; cuenta con 1560 habitantes y su principal fuente de trabajo es la agricultura y la ganadería. En $48 \%$ de las familias, al menos uno de sus miembros contaba con experiencia migratoria hacia Estados Unidos.

Villa Morelos se ubica en una región de montaña situada al sur-oeste de la ciudad de Guadalajara; pertenece al municipio de La Manzanilla de la Paz; cuenta con una población aproximada de 1600 habitantes; entre sus principales fuentes de ingresos se encuentran la ganadería, la elaboración de productos derivados de la leche, la agricultura y la pesca para autoconsumo. En $34 \%$ de las familias al menos un miembro había emigrado a Estados Unidos. 


\section{Aproximación a los informantes}

La estrategia de acercamiento hacia los participantes fue la misma en las poblaciones mexicanas. Con el apoyo de algunos miembros de estas comunidades, previamente contactados, se convocó a una reunión informal a personas con experiencia migratoria para que conocieran al equipo de trabajo y los objetivos del estudio.

En Estados Unidos se trabajó en dos localidades del estado de Texas: Longview y El Paso. En la primera se contactó previamente con informantes clave, conocidos con anterioridad en México; éstos contactaron a los posibles participantes para ser invitados e informados del propósito de la investigación: algunos se visitaron en sus propias viviendas y otros en una reunión informal realizada en casa de uno de ellos.

Aun cuando se les comentó que podrían recibir un incentivo económico por su participación, los informantes declinaron su aceptación y decidieron destinarla a los gastos que generó la reunión.

En El Paso, se estableció contacto previo con autoridades del albergue de trabajadores del campo Centro Sin Fronteras, sitio a donde acuden los trabajadores migrantes con el propósito de ser contratados por el empleador, generalmente para trabajos agrícolas y, sobre todo, en la recolección de hortalizas.

Se invitó a participar en el estudio, de forma voluntaria, a algunos trabajadores; los que aceptaron recibieron 20 dólares como compensación por su participación.

\section{Proceso de selección de los participantes}

En la selección de los participantes se utilizó la técnica de muestreo propositivo (Arcury y Quandt, 1998), con los siguientes criterios de inclusión: hombres, mexicanos, mayores de 18 años de edad, con experiencia migratoria de por lo menos un año, con residencia, al momento del estudio, en Estados Unidos o que hubiesen regresado a México.

Participaron en el estudio general 198 hombres migrantes (104 en México y 94 en Estados Unidos). En la fase cualitativa se incluyó a 38 participantes: 19 de México (10 de San Antonio de los Vázquez y 9 de Villa 
Morelos) y 19 de Estados Unidos (13 de Longview y 6 de El Paso). La edad promedio de los participantes en México fue de 49 años y en Estados Unidos de 51. De los participantes, 28 (73\%) reportaron ser casados o vivir en unión libre, 35 (92\%) practicar la religión católica, 2 (6\%) no recibieron educación formal, $21(56 \%)$ cursaron entre 1 y 6 años de escuela, 9 (23\%) entre 7 y 12 años y 6 (15\%) más de 12 años. De su actividad laboral, $23(61 \%)$ trabajaban en el área agrícola, principalmente en el campo con actividades de siembra y recolección de frutos y hortalizas, 8 (20\%) en la industria de la construcción, $4(10 \%)$ en servicios (ayudante de cocina, jardinero, cuidar y asear casas) y 4 (9\%) reportaron ser jubilados. Los participantes en Estados Unidos reportaron como lugares de procedencia algunas localidades de los estados mexicanos de Querétaro, Michoacán, San Luis Potosí, Tamaulipas y Nuevo León.

\section{Recolección de la información}

La información se obtuvo por medio de entrevistas a profundidad (Mella, 2003, pp. 147-174), mediante las cuales se exploraron los siguientes temas: proceso migratorio, experiencia sobre el cruce de la frontera, estados emocionales antes, durante y después de la experiencia migratoria, experiencia laboral en Estados Unidos, consumo de alcohol y drogas por iniciativa propia y por invitación de otros migrantes. Para los que retornaron a México, se agregó la exploración de motivos del regreso. Las entrevistas se realizaron en los hogares de los participantes de las localidades de México; en Longview, unas se realizaron en las casas de los participantes y otras en el salón de una iglesia; en El Paso, las entrevistas se realizaron en aulas del albergue Centro Sin Fronteras. En todos los casos se verificó la privacidad de los espacios antes de iniciar la entrevista, sólo estaban presentes el participante y un investigador. Se cuidó que todos los participantes leyeran y firmaran los formularios de consentimiento informado, proporcionados por la Junta de Revisión Institucional de la UTEP y la U de G; el formulario fue redactado en idioma inglés y español (como lo marcan los lineamientos de la junta binacional), lo que evitó algún problema para los participantes; todos aceptaron firmarlo en forma voluntaria, sin hacer cuestionamientos al mismo. 


\section{Análisis de la información}

Las sesiones de entrevista tuvieron una duración entre 45 minutos y dos horas y media. Fueron audiograbadas y transcritas en un procesador de texto. Para el análisis de la información se utilizó el método de análisis fenomenológico que establece las siguientes etapas: previa o clarificación de los presupuestos (descritos en la introducción), descriptiva u obtención de la información (descrita en este apartado) y estructural.

La etapa estructural incluyó:

- Lectura general de la descripción de la información.

- Delimitación de las unidades temáticas naturales: 1648 citas textuales, agrupadas en 46 códigos temáticos, formando con ello el corpus de análisis.

- Determinación del tema central que domina cada unidad temática: Se identificaron cinco temas o categorías centrales:

a) Proceso migratorio: Con las experiencias implicadas en el tránsito hacia Estados Unidos y la estancia en este país, y, en su caso, las de retorno a México. Incluyó, también, motivaciones para migrar, inicio del proceso migratorio, experiencia en el cruce de la frontera (forma, lugar de cruce y peligros), lugares a los que migró, redes de apoyo migratorio, migración con la familia, la "migra", deportación y situación legal, y motivos para no quedarse en Estados Unidos.

b) Estados emocionales: Con las descripciones de las experiencias de diversos estados de ánimo, vividos durante el tránsito y estancia en Estados Unidos y, en su caso, los de retorno a México. Incluyó, además, sentimientos al momento de cruzar la frontera, estados de ánimo en Estados Unidos y en México, desarrollo de actividades recreativas en aquel país y en México, convivencia diaria en Estados Unidos y en México, relaciones sentimentales allá y en México, y redes de apoyo emocional.

c) Consumo de alcohol y drogas: Describe la experiencia en el consumo de alcohol y drogas. Ahí se incluyeron consumo de sustancias en Estados Unidos y en México, motivaciones para el uso de sustancias, percepción del uso de sustancias en general, por iniciativa 
propia y por invitación de otras personas, en México y en Estados Unidos, y rechazo al consumo de sustancias.

d) Actividad laboral: Describe el desarrollo de actividades remuneradas, en México y Estados Unidos. Abarca la búsqueda de trabajo, entorno de trabajo en aquel país y en México, experiencia laboral allá y en México, derechos laborales, discriminación y riesgos laborales.

e) Apoyo social: Describe el apoyo recibido durante la migración. Éste contiene diferentes tipos o formas de apoyo, así como diversos componentes que lo constituyen.

Los resultados se presentan para el tema central, en lenguaje científico y, en la integración de los temas centrales, se utiliza una estructura descriptiva que facilita la composición de todas las estructuras particulares hacia una estructura general (Martínez, 2006, pp. 167-188). Para el proceso de análisis se utilizó el software Atlas.ti. v6 (Muhr, 1997).

\section{Hallazgos}

Las emociones durante la migración: Entre el miedo y la nostalgia

Los informantes entrevistados, en ambos lados de la frontera México-Estados Unidos, hicieron referencia a diversos estados emocionales vivenciados en las diferentes fases de su experiencia migratoria: antes de iniciar el viaje, durante el cruce de la frontera, su estancia en aquel país y, en su caso, el retorno a México.

\section{Las emociones antes de iniciar el viaje}

En los relatos de los migrantes, ninguno hizo referencia a las emociones en el periodo de preparación del viaje.

Las emociones durante el tránsito de México a Estados Unidos de América

Durante el cruce de la frontera se vivió nostalgia, miedo, dolor, incertidumbre. Nostalgia por alejarse de casa, por extrañar a los seres queridos, 
por el temor a no ver más a la familia, por la soledad. Miedo ante la posibilidad de ser descubiertos, de morir, de entrar a un país desconocido, ser deportados a México, no tener dinero para intentar de nuevo el cruce o regresar a su comunidad de origen, a los peligros del desierto. Un hombre entrevistado en Jalisco refirió:

Me empezaban a llegar más los nervios porque mi primo empezaba a hablar que por donde pasábamos se oían muchas víboras... que había un viboral. iHijo de su madre... y a ver si no nos pica alguna! Además, hay muchas arañas por el desierto.

Dolor por la muerte de algún compañero de viaje. Incertidumbre ante la inseguridad de ver nuevamente a su familia. Un hombre de Jalisco mencionó:

En ese transcurso de casi dos días, yo ya extrañaba a mis papás. Estaba todavía chavalo, extrañaba a mis papás, a mi novia; yo decía: "Se va a quedar sola... se la va a llevar otro". Era lo que más me hacía que me sintiera mal, en el momento que decía: ¿̇Iré a regresar o no?

\section{Emociones durante la estancia en Estados Unidos}

Durante su estancia en Estados Unidos sufrieron preocupación, tristeza, nostalgia, depresión, estrés, incertidumbre, confusión, soledad y miedo. Preocupación por la familia que se quedaba en México, por obtener o conservar un trabajo. Tristeza cuando les comunicaban la muerte de algún familiar en México y se veían impedidos de regresar por su condición de ilegales o por la lejanía de sus familias. Nostalgia al recordar el lugar de procedencia, sus costumbres, la comida, las actividades que realizaban con sus compañeros, el recuerdo de la convivencia en la comunidad; añoraban a los padres o a las esposas. Uno de ellos comentó que llegó con su familia cuando tenía ocho años, pero el tipo de vida que experimentó no le gustó; él y sus hermanos estaban siempre solos, "presos" en el departamento donde vivían, sólo salían los fines de semana con sus padres para hacer compras. Otro dijo que su padre lo golpeaba todo el tiempo, por lo cual prefería estar en la calle. Uno más comentó: 
Allá, uno en veces que amanece uno de malas, con nostalgia, no sé cómo le nombran, como enfadado, ahí como malhumorado, yo sentía como mal humor, que me hablaran así, no diario... nada más que me empezara a acordar yo de acá... y ya me sentía yo como mal... y ya otro día amanecía bien, con ganas de ir a trabajar.

También se percibió añoranza por los días soleados en su pueblo; algunos vivieron en lugares donde los rayos del sol eran escasos.

La depresión fue otra emoción de los migrantes durante su estancia en Estados Unidos: no tener con quien hablar o apoyarse ante algunos problemas que debían enfrentar. Un hombre en Texas dijo:

No le echo la culpa a nadie, quiere andar uno acá, pero de todos modos la soledad es el pior enemigo del ser humano.

El estrés frente a las diferencias culturales, las situaciones laborales, el contacto con grupos culturales considerados hostiles (afroamericanos y méxicano-americanos) también fue una emoción de los migrantes. Las diferencias culturales entre sus pueblos de origen y las de Estados Unidos generó problemas de comunicación con personas de otras culturas (estadunidense, méxico-americana y afro-americana), lo que implicó no socializar con ellas. Además, identificaron que algunas culturas, especialmente las pertenecientes a los grupos méxicano-americanos y afro-americanos, mostraron ser violentas y de difícil convivencia; estas experiencias las tuvieron cuando vivían en barrios donde se establecían bandas juveniles que les exigían, especialmente a las personas que migraron muy jóvenes a Estados Unidos, realizar alguna iniciación (como pelearse o golpear a alguien) para ser parte del barrio o de la banda y, con ello, lograr no ser molestados.

La incertidumbre de no saber cuándo verían nuevamente a su familia y no ver crecer a sus hijos fueron emociones durante su estancia en Estados Unidos. La confusión que enfrentaban algunos de ellos, que tenían varios años viviendo en el país del norte, cuando visitaban ocasionalmente a sus comunidades de origen: regresaban porque extrañaban a su país y les daba gusto ver a su familia y amigos, sin embargo, cuando llegaban a su comunidad se sentían extraños, ya que poco conocían a las personas. Un hombre en Texas dijo: 
La verdad que ya no tengo amigos en México, porque todos se vinieron. Y últimamente que voy, me paseo ahí en el jardín y miro puras caras desconocidas; a lo mejor ellos piensan que yo soy desconocido, pero ya no los conozco. Me siento extraño cuando voy.

Las emociones de retorno a México

De regreso a México, se experimentó satisfacción, incertidumbre, tristeza. Satisfacción al estar con la familia, grupo social y cultura. Incertidumbre ante la situación económica que enfrentaban por la escases de trabajo o por trabajos mal remunerados. Tristeza frente a la separación de familiares que aún vivían en el "otro lado".

\section{Actividad laboral}

El trabajo en Estados Unidos provoca presión frente a la exigencia de puntualidad y productividad (situación más laxa en México); un hombre de Jalisco indicó:

Allá, si quieres mantener tu trabajo tienes que ser constante, que a las ocho, a las ocho, no a las ocho cinco. Te la pasarán una vez, pero ya la siguiente vez sabes qué: "vete y agarra otro trabajo".

Mencionaron que no platicaban con sus compañeros en el espacio laboral, ya que los supervisores les llamaban la atención e incluso podían perder su trabajo por esta causa. Un hombre de Jalisco comentó:

Si estás trabajando poco los miras... a veces nomás entra uno y sales, está uno cerquitas y duras un año sin mirarse, o nomás de pasadita, porque cada quien tiene su trabajo.

Aunque, para otros, las exigencias de puntualidad y productividad les llevó a desarrollar la disciplina laboral. Desde esta perspectiva, lo que para unos es fuente de estrés, en otros constituye una forma de desarrollar nuevas habilidades ante condiciones de trabajo diferentes. 
Consumo de sustancias: Cerveza social vs. drogas dañinas

Los participantes señalaron percibir una imagen negativa de Estados Unidos en lo referente al consumo de drogas: visualizan un espacio con mayor consumo y acceso a sustancias ilegales. La imagen de México fue diferente: anteriormente el consumo no tenía mucha difusión, sin embargo ahora está en proceso de imitar a Estados Unidos. Entre las razones que se citaron para la existencia en Estados Unidos de un mayor consumo de sustancias están las del tipo económico; en México no se tienen ingresos suficientes para comer, menos aún para comprar una cerveza; mientras que en el país vecino se gana más dinero y por ello pueden darse el gusto de comprar alcohol y drogas.

Manifestaron una visión negativa acerca del consumo de drogas (con pocas posibilidades de recuperación), sin embargo, mostraron más aceptación sobre el consumo de alcohol (tiene "mayores posibilidades de recuperación", según un informante de Jalisco). El alcohol y las drogas se consideran productos caros que afectan la economía familiar. Las drogas se consideran dañinas para la salud, adictivas y difícil para dejar de consumirlas (un hombre de Jalisco dijo: "el alcohol se puede controlar y las drogas ya no").

Los motivos para el consumo de alcohol o drogas fueron la necesidad de lograr una mayor resistencia durante el cruce de la frontera y en el trabajo; para olvidar problemas que generan soledad, depresión e incertidumbre; para obtener seguridad y tranquilidad; para adaptarse al grupo de amigos; tomaban alcohol para evitar drogarse, o bien, usaban drogas para "cortar" el estado de embriaguez. Otros motivos fueron de tipo familiar (antecedentes de parientes alcohólicos o para huir de problemas familiares), también por curiosidad, gusto y creencias ("el agua hace daño", "la mariguana es medicinal"), aunque gran parte de los entrevistados indicó que es una decisión personal comenzar el consumo de alcohol y las drogas. Algunos comentaron que con frecuencia convivían con personas que usaban drogas, sin embargo, decidieron no unirse al grupo en dicha actividad. Un hombre en Texas opinó:

El que quiere lo hace, y el que no, no. Yo nunca he hecho eso. A mí, si me dan mariguana la dejo; si me ofrecen cocaína tampoco la quiero, para nada, no me gustan esas cosas. 
La mayoría de los participantes reconoció experimentar diferentes patrones en el consumo de sustancias antes y después de su experiencia migratoria. En la etapa previa a la migración tenían un consumo moderado de alcohol, de tipo social, como convivencia de fines de semana. Durante su estancia en Estados Unidos, se intensificó el consumo, agravado por el más fácil acceso a ellas y por la mejoría de sus condiciones materiales. Las drogas que consumieron en ese país fueron marihuana, ácidos, crack (piedra), cocaína y cristal (ubicaron a la mariguana en primer lugar), motivados por la influencia de amigos o compañeros de trabajo. Las razones para consumirlas fueron diversas, entre las que destacan: olvidar los problemas y a la familia en México, rendir en el trabajo, el gusto de probarlas, por soledad, por no tener trabajo, por convivencia. Un hombre de Jalisco dijo al respecto:

La mariguana no [la consumía] diario, pero cada ocho días sí. Los amigos fumaban y me decían: "cálale", y yo, pues sí le fumaba a esa cosa y me sentía a gusto, relajado, como que se me olvidaban los problemas.

Los informantes con mucho tiempo en Estados Unidos reportaron abandono o disminución en el consumo de alcohol o drogas por alguna de las siguientes razones: dar buen ejemplo a sus hijos, por decisión personal, les generaba altos costos y daños a la salud ("las drogas matan el cerebro", "el alcohol embrutece”), desempeñarse mejor en el deporte, por el estricto control en su espacio laboral, para evitar problemas policiacos y observar muerte por sobredosis. La mayoría de los migrantes que regresaron a México dijeron que, aunque aún consumían alcohol o tabaco, habían disminuido el consumo que tenían en Estados Unidos; entre las razones de esta disminución están: no tener suficiente dinero para ello, ser caro, generar daños a la salud, evitar que la familia los encontrara consumiendo y evitar dar mal ejemplo a los hijos. Un entrevistado en Jalisco dijo:

Aquí ya no me gusta. Porque aquí es muy caro todo eso. Y porque mi esposa me va a regañar si me ve fumando. Mi hijo también me va a decir que por qué ando en eso. Y no quiero darle ese ejemplo.

Sólo uno comentó que continuaba fumando mariguana, ya que se sentía en casa. 
Las personas entrevistadas en el albergue para trabajadores agrícolas de El Paso presentaron mayor resistencia para hablar del tema. Reconocieron que tomaban cerveza, pero que lo hacían solamente al exterior de las instalaciones del albergue, ya que no les permitían ingresar con aliento alcohólico o en estado de embriaguez, y estaba prohibido ingerir cervezas dentro del mismo. Dijeron que tomaban cerveza sólo los fines de semana; los menos reportaron que tomaban diario, pero en "pocas cantidades" ( 2 a 4 por día). La cantidad que consumían los fines de semana fluctuaba entre 6 y 15 cervezas por persona; mientras que en un consumo grupal se consumían hasta 8 cartones (aproximadamente 160 cervezas) entre 10 personas (en promedio). Uno de los motivos para este consumo fue principalmente el clima cálido, sobre todo en el lugar de trabajo. Así, una forma de hidratación y recuperación del cansancio era bebiendo cerveza, ya que "tomar agua hace daño". Algunos mencionaron que bebían porque se sentían solos, por problemas o por el ejemplo que les dieron sus padres alcohólicos. Sin embargo, la mayoría comentó hacerlo por gusto y diversión; para uno de ellos era un hobbie. Un hombre de Estados Unidos que usaba drogas, pero no se consideró adicto, refirió:

Yo a veces usaba drogas como para no dormirme, pero sería una vez al mes; a veces que tenía sueño, o algo, las usaba para despertar, pero [yo] no era adicto.

Los efectos del uso de estas sustancias fueron: sentirse seguros y "más hombres" o "más machos" y no dormirse en el trabajo.

\section{Fuentes de apoyo emocional y social: familia, amigos, religión}

Pareciera que el apoyo social, informal, proveniente de las redes sociales al tiempo de llegada y durante su estancia, puede ser, para los migrantes, un importante amortiguador del estrés. Un hallazgo interesante fue el señalamiento de espacios y personas donde obtenían apoyo emocional positivo: familia, amigos e iglesia.

Los informantes que vivían con su pareja, o familia, reportaron estados emocionales satisfactorios, así como un mayor control sobre el consumo de alcohol, incluso se identificó la abstinencia total. Al arribar a Estados Unidos, el principal apoyo emocional que tuvieron los migrantes fue de sus 
familiares y luego los amigos. En lo general tenían parientes ya instalados en comunidades de ese país, lo que facilitó su adaptación. Algunos refirieron que la familia era muy importante para ellos y lo que les había dado la fuerza para salir adelante, sobre todo si estaban unidos. Un hombre en Texas comentó:

Me ayudó mucho tener a mi familia... tener esa confianza de que alguien te diga en casa: "Tú puedes, tú puedes". Mi madre siempre tuvo eso conmigo: "Ustedes pueden".

La mayoría de los migrantes entrevistados reportaron estar casados (antes de salir de México), además de vivir con la esposa y los hijos en Estados Unidos. Quienes migraron muy jóvenes encontraron pareja en Estados Unidos y vivían en unión libre; éstos comentaron que tener a alguien en quien apoyarse era muy importante para salir adelante. Así, el papel de la familia se constituyó como una de las principales redes de apoyo.

La ausencia de una red familiar de apoyo se observó entre los migrantes entrevistados en el albergue de trabajadores agrícolas; ellos comentaron preferir vivir en ese sitio a vivir solos. Ahí se apoyaban en sus problemas a través de la conversación; uno de ellos expresó: "Aquí de perdida mira uno ahí a la gente... aunque a veces se caiga uno gordo"; no obstante de que la convivencia sólo con hombres tuviera sus dificultades, ya que entre ellos se da poco la comunicación. Un hombre en Texas dijo al respecto:

Los mexicanos, más que los hispanos, tienen esa idea en la cabeza, de macho, y en la realidad son diferentes, los hombres a veces no aceptan consejos entre hombres. Yo viví con amigos donde a nadie le importaba lo del otro; o sea, ellos no sabían si me sentía deprimido, no les importaba.

Habría que sumar lo anterior a que en el ambiente laboral no les era permitido conversar con los compañeros de trabajo. Esto implicaba cambios en las formas de socializar dentro de los ambientes laborales; en México son más permisivos y se admite el libre intercambio de ideas, puntos de vista e incluso de emociones.

Otras redes de apoyo se formaban en las relaciones con amigos. La amistad se constituía, así, como un valor indispensable para la convivencia, 
la formación de redes laborales, el contacto con la cultura de origen y el desarrollo de actividades deportivas. En los días de descanso, los informantes con familia reportaron que asistían a misa y algunos jugaban futbol (comentaron que de jóvenes practicaban todo el tiempo). También realizaban reuniones con sus amigos, donde comían y bebían. Otros sabían tocar instrumentos musicales y formaban parte de los coros religiosos o las bandas que tocaban en fiestas; lo hacían más como una forma de distracción que como un trabajo.

Los informantes del albergue de trabajadores agrícolas reportaron que los fines de semana regresaban a México (a Ciudad Juárez) para pasear en las plazas, ver a las muchachas y tomar cerveza, aunque esto sólo lo hacían quienes contaban con identificación de residente en Estados Unidos. Estas actividades eran para distraer sus sentimientos de tristeza, ansiedad y nostalgia y así no pensar en la familia que estaba lejos. Por lo que esta población migrante se encontraba en estado de mayor vulnerabilidad para desarrollar depresión u otros problemas mentales. Sin embargo, esta condición pudiera ser menor frente a la de los migrantes que vivían completamente solos.

La asistencia y participación en rituales religiosos se identificó como un espacio importante para los migrantes; en la iglesia obtenían consejería espiritual y emocional, apoyo legal y convivencia en actividades religiosas y recreativas. Algunos relataron que el acercamiento a la iglesia les ayudó en los momentos difíciles de su estancia en Estados Unidos, incluso les facilitó dejar de consumir bebidas alcohólicas y ser más responsables con su familia. Uno de los entrevistados en Texas dijo al respecto:

Me ayudó acercarme más a la iglesia, para empezar, cuando me casé me ayudó algo, pero pienso que a mí lo que más me ayudó fue la iglesia. Siempre he querido avanzar, o sea, yo siempre tomo los días como van viniendo.

Además, fue un apoyo en los momentos de soledad, para encontrar una salida y la fuerza para continuar. $\mathrm{Al}$ respecto, un hombre comentó:

Mis primos en Longview asistían a la iglesia y me invitaron, pero yo no quería nada con la iglesia; cuando estaba en ese tiempo, de depresivo, me invitaron a ayudarles a dirigir la misa por las bodas, y ahí había dinero, entonces yo les 
ayudaba, y empecé a ir a la iglesia por la música, mi vida empezó a cambiar, mi relación con la pareja y mi relación conmigo mismo.

\section{Discusión}

A partir de la experiencia migratoria se logró identificar el estado de vulnerabilidad física y emocional que viven los migrantes durante la fase del cruce de la frontera, mismo que parece coincidir con lo reportado por Salgado (1996).

Las tensiones acumuladas durante la estancia en Estados Unidos pueden tener serios efectos para la salud de los migrantes, como lo señaló Bhurga (2004). Las tensiones, identificadas en el grupo abordado, parecen relacionarse con las diferencias culturales en Estados Unidos que son fuente de estrés y presión social, como las situaciones laborales y el contacto con grupos culturales considerados hostiles (afroa-mericanos y mexicano-americanos).

Los migrantes, en el ambiente de trabajo, viven experiencias que parecen más demandantes que las vividas en sus contextos de origen; no sólo por la situación de puntualidad y productividad, sino por el aislamiento y la amenaza constante de la posible pérdida del trabajo. Esto también se ha identificado en otros grupos, como lo ha reportado Sánchez y sus colaboradores (2006). La situación se agrava ante las dificultades que los migrantes reportan respecto de lograr una buena comunicación con los compañeros de trabajo y con las personas con las cuales conviven cotidianamente, sobre todo en la expresión de sus emociones. Lo anterior está relacionado con los elementos de la identidad masculina (Ramírez, 2005, pp. 47-48), donde la expresión de emociones "negativas" está asociada al género femenino.

La imagen diferente a la propia de los migrantes mexicanos respecto de la población mexicano-americana puede relacionarse con lo citado por Bhugra respecto de que, bajo ciertas circunstancias, el apoyo social de un mismo grupo disminuye por causa del estigma, lo que puede asociarse a problemas de salud mental (Bhugra, 2004).

A los migrantes que regresan por temporadas cortas a sus comunidades de origen les sucede que los acontecimientos en torno a su estancia los hacen sentirse como extraños, por no conocer a otros o no ser reconocidos 
ellos; esto podría tener relación con los cambios identitarios que se generan con el proceso de ajuste frente a una cultura diferente de la propia. Ya que la identidad de una persona emerge y se reafirma sólo en la confrontación con otras identidades, en el proceso de interacción social, para los migrantes resulta cuestionada su pertenencia social, por no sentirse identificados con el grupo de personas que habitan en sus comunidades de origen. Sin embargo, los fenómenos de aculturación o de transculturación no implican, automáticamente, una pérdida de identidad, sino sólo su recomposición adaptativa (Giménez, 2004).

Las vivencias de los migrantes, del grupo estudiado, en torno a los procesos personales de consumo de alcohol se correlacionan con los estudios de Borges y colaboradores (2007) y Sánchez-Huesca y colaboradores (2006), respecto de que el consumo es menor antes de migrar a los Estados Unidos. La imagen del alcohol es menos negativa ante otras drogas, ya que se puede controlar su consumo, además de que coadyuva a no drogarse, a hidratarse, a recuperarse del cansancio, a sentir menos lacerante la soledad, aunque también se consume por gusto, como una forma de diversión.

Para el consumo de otras sustancias diferentes al alcohol, en el grupo abordado se identificaron elementos coincidentes con lo reportado por Borges y colaboradores (2007) y Sánchez-Huesca y colaboradores (2006). A este tipo de sustancias se les connota como drogas dañinas, más accesibles en Estados Unidos que en México, adictivas, pero que permiten una mayor resistencia en trances difíciles como el cruce de la frontera, o coadyuvantes en algún problema de salud por considerarlas "medicinales”, como la marihuana, o para evitar el sueño cuando así se requiere, especialmente en la jornada de trabajo.

Se ha identificado que los efectos de la migración en la salud mental de los individuos pueden ser positivos o negativos dependiendo de determinados factores. Al parecer el apoyo social informal, proveniente de las redes sociales al tiempo de llegada y durante la estancia, beneficia a los migrantes en su salud mental ya que se convierte en un importante amortiguador del estrés y, con ello, se evita no sólo la depresión sino, también, otros tipos de trastornos (McKay, Macintyre y Ellaway, 2003, pp. 12-13). Así, un hallazgo interesante en el estudio fue el señalamiento de espacios y personas de donde obtenían apoyo emocional positivo, como la familia, los amigos y la iglesia. 
La actividad religiosa fue importante en la identidad cultural de poblaciones migrantes, situación que ha sido reconocida por autores como Rivera (2006). Así, según lo encontrado, las iglesias constituyen un importante espacio de convivencia entre migrantes, para el apoyo emocional, espiritual e incluso legal.

La convivencia con los paisanos permite también espacios de recreación y, en algunos casos, involucrarse con sus comunidades de origen; como lo han demostrado los clubes de migrantes que impulsan un tipo de desarrollo socioparticipativo que rescata la noción de desarrollo social antes que la de desarrollo económico (Hernández, 2006).

De acuerdo con los hallazgos, concluimos que las condiciones que facilitan la vivencia de emociones negativas en la población migrante y que pudieran conducirlos al consumo de alcohol y drogas son: la distancia física y emocional con la pareja o la familia, las presiones laborales, la amenaza de deportación, la dificultad para expresar las emociones, el ambiente social, la dificultad para la integración a la nueva cultura, que pudiera convertirse en un sustrato de "arenas movedizas" para caer en la depresión. Sin dejar de lado algunos facilitadores para el acceso al consumo de alcohol y drogas como amigos o situaciones económicas.

Frente a esta realidad, creemos que se debe trabajar en la construcción e identificación de espacios que posibiliten a los migrantes algún tipo de apoyo emocional, legal, psicológico, de reunificación familiar, desarrollo de convivencias, actividades recreativas, aprendizaje del idioma inglés, conservación de la cultura de origen y socialización de la nueva cultura.

Aunque reconocemos que las limitaciones de este estudio se encuentran en la no inclusión del punto de vista de otros miembros de la familia de los entrevistados, consideramos que para futuros trabajos sería recomendable incluirlos, tanto los que viven con ellos en Estados Unidos como los que se quedan en México.

\section{Bibliografía}

Arcury, T. y Quandt, S. (1998). Qualitative methods in arthritis research: Sampling and data analysis. Qualitative Methods, 11(1), 66-74. 
Bhugra, D. (2004). Migration and mental health. Acta Psychiatr Scand, 109, 243258.

Borges G., Medina-Mora, M. E., Breslau, J. y Aguilar-Gaxiola, S. (2007). The effect of migration to the United States on substance use disorders among returned Mexican migrants and families of migrant. American Journal of Public Health, 97(10), 1847-1851.

Comisión de Salud Fronteriza México-Estados Unidos. (2003). Frontera saludable 2010. Una agenda para mejorar la salud en la frontera México-Estados Unidos. Resumen Ejecutivo de la Comisión de Salud Fronteriza México-Estados Unidos. Recuperado de <http://www.paho.org/hia/archivosvol2/paisesesp/Frontera\%20 de\%20Estados\%20Unidos\%20y\%20México\%20Spanish.pdf $>$.

García, V. y Gondolf, E. (2004). Transnational Mexican farm workers and problem drinking: A review of the literature. Contemporary Drug Problems, 31(1), 129-161.

Giménez, G. (2004). Materiales para una teoría de las identidades sociales. En J. M. Valenzuela (coord.). Decadencia y auge de las identidades. Tijuana, México: El Colegio de la Frontera Norte, Plaza y Valdés.

Hernández, L. (2006). ¿De aquí p’a allá o de allá p’a acá? Clubes de migrantes jaliscienses: Promoción estratégica de capital social y desarrollo. Migraciones Internacionales, 3(4), 60-84.

Holmes, S. (2006). An ethnographic study of the social context of migrant health in the United States. PLosmed, 3(10), 1776-1793. Recuperado de <http://www. plosmedicine.org/article/info:doi/10.1371/journal.pmed.0030448>.

McKay, L., Macintyre, S. y Ellaway, A. (2003). Migration and health: A review of the international literature. Documento occasional. MRC. Medical Research Council, Glasgow, Social \& Public Health Sciences Unit. Recuperado de $<$ http://www.sphsu.mrc.ac.uk/library/occasional/OP012.pdf $>$.

Maffesoli, M. (1997). Elogio de la razón sensible. Una visión intuitiva del mundo contemporáneo. Barcelona: Paidós.

Martínez, M. (2006). Comportamiento humano (2da. ed.). México: Trillas.

Mella, O. (2003). Metodología cualitativa en ciencias sociales y educación. Santiago: Primus.

Muhr, T. (1997). Scientific software development's aTLAS-ti. Visual qualitative data. Versión 6. Berlín: Scientific Software Development.

ops (Organización Panamericana de la Salud). (2007). Frontera de Estados Unidos y México. Salud en las Américas, 2007, Autor. Recuperado de <http:// www.paho.org/hia/archivosvol2/paisesesp/Frontera\%20de\%20Estados\%20 Unidos\%20y\%20México\%20Spanish.pdf >.

ops (Organización Panamericana de la Salud). (2009). Epidemiología del uso de drogas en América Latina y el Caribe: Un enfoque de salud pública. Autor. Recuperado de < http://new.paho.org/hq/dmdocuments/2009/epidemiologia_ drogas_web.pdf $>$. 
Ramírez, J. C. (2005). Madejas entreveradas. Violencia, masculinidad y poder. México: Plaza y Valdés.

Rivera, L. (2006). Cuando los santos también migran: Conflictos transnacionales por el espacio y la pertenencia. Migraciones Internacionales, 3(4), 35-59.

Rojas, E., Fleiz, C., Villatoro, J., Gutiérrez, M. L. y Medina-Mora, M. E. (2009). Tendencias del consumo de drogas de 1998 a 2005 en tres ciudades de la zona norte de México: Ciudad Juárez, Monterrey y Tijuana. Salud Mental, 32(1), 13-19.

Salgado, N. (1996). Problemas psicosociales de la migración internacional. Salud Mental, (19), 53-59.

Salgado, N. (2002). Research and clinical perspectives on Mexican migration: Those who go, those who stay. Journal of Multicultural Nursing and Health, 8(2), 23-31.

Sánchez-Huesca, R., Arellanez-Hernández, J. L., Pérez-Islas, V., Rodríguez-Kuri, S. E. (2006). Estudio de la relación entre consumo de drogas y migración a la frontera norte de México y Estados Unidos. Salud Mental, 29(1), 35- 43.

Villatoro-Velázquez, J. A., Medina-Mora, M. E., Fleiz-Bautista, C., Téllez-Rojo, M. M., Mendoza-Alvarado, L. R., Romero-Martínez, M., Gutiérrez-Reyes, J. P. et al. (2011). Encuesta Nacional de Adicciones 2011: Reporte de drogas. Recuperado de <http://www.conadic.salud.gob.mx/pdfs/ENA_2011_DROGAS_ILICITAS_.pdf > . 